\title{
Article \\ A Case Study on Remote Instrumentation of Vibration and Temperature in Bearing Housings
}

\author{
Mariana Cardona ${ }^{1}$, Michael Cifuentes ${ }^{2}$, Byron Hernandez ${ }^{3, *}$ and William Prado ${ }^{1, *}$ \\ 1 Department of Mecathronics Engineering, Universidad Tecnológica de Pereira, \\ Pereira 66001, Risaralda, Colombia; marianacarto_94@utp.edu.co \\ 2 Department of Electrical Engineering, Universidad Tecnológica de Pereira, \\ Pereira 66001, Risaralda, Colombia; mcifuentes@utp.edu.co \\ 3 Department of Electrical and Computer Engineering, Marquette University, Milwaukee, WI 53233, USA \\ * Correspondence: byron.hernandez@marquette.edu (B.H.); prado@utp.edu.co (W.P.)
}

Citation: Cardona, M.; Cifuentes, M.; Hernandez, B.; Prado, W. A Case Study on Remote Instrumentation of Vibration and Temperature in Bearing Housings. J. Low Power Electron. Appl. 2021, 11, 44. https://doi.org/ 10.3390/jlpea11040044

Academic Editor: Andrea Acquaviva

Received: 13 August 2021

Accepted: 16 November 2021

Published: 19 November 2021

Publisher's Note: MDPI stays neutral with regard to jurisdictional claims in published maps and institutional affiliations.

Copyright: (c) 2021 by the authors. Licensee MDPI, Basel, Switzerland. This article is an open access article distributed under the terms and conditions of the Creative Commons Attribution (CC BY) license (https:// creativecommons.org/licenses/by/ $4.0 /)$.

\begin{abstract}
Data collection is one of the most relevant topics of modern automation and industry. It is usually a costly and time-consuming task, especially in continuous processes. Our case study takes place in a sugar cane mill. The required continuous operation of a belt conveyor for bagasse transportation makes it a critical system in the overall production process. Therefore, a predictive maintenance tool is highly applicable here. We identified bearing housings as critical points for data collection intended for prognostics of the conveyor. However, given the number of points, the cost of a commercial solution becomes unfeasible by our company. This paper reports the development of low-cost devices for measurements and wireless transmission of vibration and temperature data from bearing housings. We assessed several sensor options and made decisions based on a cost-suitability commitment, which led to the design of the electronic devices. The devices were tested for correct operation, reliability (99\%), and relative measurement errors under $1.2 \%$. From the tests, we conclude that our proposal is appropriate for our case study's industrial needs and budget restrictions.
\end{abstract}

Keywords: sensing network; LoRa; machinery prognostics; belt conveyor

\section{Introduction}

Maintenance is one of the most important topics for a reliable and long lifecycle of industrial equipment. There are several maintenance methodology approaches for operational cost and life-use optimization. The latest and probably the most effective approach long-term is predictive maintenance. Accurate and reliable data measurement and acquisition to predict failure events is the general goal of this approach. The use of measurement systems for data collection and pattern analysis has shown promising applicability in this field [1,2].

To achieve efficient maintenance, prognostics is essential for task scheduling that reduces operating costs and improves productivity. A prognostic is an estimation of the current condition of the target system; this estimation is based on several models, including past event information, real-time observations, life-use (LU), and operational models. In general, all of these appraisal models are fitted, adjusted, and based on data, i.e., information collected from measurement systems located on the equipment. Thus, the quality of the predictions is highly dependent on the quality of the data.

In Colombia, industry is foraying its transformation towards Industry 4.0. which entails the enforcement of high-end technology for both production and maintenance tasks. However, some technologies remain unaffordable for certain small and midsize enterprises.

Specifically, our case study takes place in a sugar cane mill. A belt conveyor there needs to operate continuously since it carries bagasse to a steam boiler for electricity generation. Furthermore, it needs to keep low down-times, and failures appear sometimes on bearing, rollers, and other moving parts. Given the above, variables such as temperature 
and vibration are critical for monitoring and analysis; nevertheless, installing standard industrial sensing networks for continuous monitoring is unsuitable for our factories and mills due to the high price of each sensor. Vibration analysis equipment, for example, costs several hundreds of dollars and can only be used in a single rolling point at a time.

Currently, the maintenance department of the sugar cane mill performs a vibration analysis once every two weeks over only two out of twelve bearings and measures temperature once each working day. The belt conveyor we studied operates $24 \mathrm{~h}$ per day every day; however, the maintenance department stores only a one-minute time window for each vibration record and manually saves the instantaneous temperature. The aforementioned implies high intervals between samples and low standardization on the measurement procedure. In this sense, the measurements are not repeatable nor reproducible and therefore are not adequate to estimate the belt conveyor condition and subsequent prognostics.

In order to improve data collection and achieve a better prognostic maintenance within a low-budget constraint, we propose a system with low-cost electronic devices, which are affordable for small and mid-size enterprises in developing countries such as Colombia. The devices need to perform the measurement in the field and transmit the chosen variables to a central receptor where a data analytic software evaluate the health of the conveyor.

We did a wide assessment of the belt conveyor looking for operating points, measurements, and communication requirements. In addition, we also developed and proposed the following items, considering the suggestions of recent studies.

- A low-cost 3-axial vibration sensor for industrial usage.

- A low-cost temperature sensor for industrial usage.

- A low-cost communication module using Long-Range (LoRa) technology for ours and similar industrial applications.

- $\quad$ A Low-Power Wide Area Network (LPWAN) operating on an industrial setup.

In order to guarantee the reliability of our devices and make an overall assessment, we executed multiple laboratory experiments, simulations, and field tests. The characterization of the conveyor for measurement conditions was aided by thermography and vibration tests. The mechanical stability, isolation, and Ingress Protection (IP) were backed by a vibrational mechanical stability assessment and Computational Fluid Dynamic (CFD) tests. Finally, the operational tests using a statistical experiment supported the operation reliability study. The results of the tests mentioned above allowed us to state the following features:

- $\quad$ Our measurement module provides vibration measurements with an uncertainty interval below $0.12 \%$ and $1 \%$ in worst-case scenarios (peaks).

- Our measurement module provides temperature measurement with an uncertainty interval below $0.5 \%$.

- Our transmission module provides a LPWAN that operates on distances up to $150 \mathrm{~m}$.

- Both uncertainty intervals and transmission assessment have a confidence interval above $99 \%$ with an estimation error below 5\%.

This paper is organized as follows: Section 2 presents a background of related works using wireless LoRa systems, instrumentation design, and measurement networks. Section 3 presents some guidelines and introduces the context to mark off the design requirements and carry out the circuitry syntheses and detailed specifications of the designed devices. Section 4 explains some experiments and operational tests carried out using the whole system. Finally, Section 5 presents a discussion of the previously mentioned experiments.

\section{Related Works}

For the proposed case study, we explored an alternative to improve the maintenance efficiency of belt conveyors. As a starting point, we considered traditional inspection methods to look for the identification of critical variables and measurement equipment. As we contemplated that the sensing system should be remote, we inquired into wireless sen- 
sors networks in industrial applications; consequently, recent breakthroughs in electronics towards the Industrial Internet of Things (IIoT) are of great interest for this project.

Vibration and acoustic signal analyses are reliable methods to diagnose the condition of belt conveyors [3]. These techniques are usually applied to bearings, pulleys, rollers, and other rotating parts while the conveyor is operating. On the other hand, belt inspection is traditionally done when the conveyor is not working. Moreover, the recent research [4] shows promising results on belt fault detection techniques for prognostics. In [5], authors used an autonomous legged inspection robot to collect acoustic signals around a belt conveyor in the mining industry; however, in the sugar cane mill, many corridors around belt conveyors are too narrow, have a slope, or do not exist. According to various academic sources, vibration analysis of bearing housings stands out for being widely used, where the preferred instrument is consistently the accelerometer [6-9].

Temperature logs are also crucial for the monitoring of machine mechanics. This statement arises from the fact that variations from the regular patterns of operating temperature are a common symptom of machinery malfunction $[3,10,11]$. There are various methods to measure temperature in industry. To begin with, familiar tools for direct contact transduction are Resistance Thermometer (RTD), thermocouple, and Negative Temperature Coefficient thermistor (NTC) sensors. However, there are no contact devices such as infrared thermometers and cameras to perform thermographic analysis $[7,8,12]$.

In addition to the variables mentioned above, numerous authors suggest alternative methods for prognostics of a belt conveyor system. The basis of the proposed techniques is the measurement of the operational parameters of the machine: time, belt speed, rotational speed, and load, among others are the most common examples. The next step is to compare the collected data with the LU cycle of the conveyor components $[7,8,13]$. Mazurkiewicz introduces in [7] the use of a magnetic field sensor, a magnet, and a pulse counter to monitor the belt conveyor integrity and other operating parameters like time, speed, and length.

The industrial revolution 4.0 relies on the use of Cyber Physical Systems (CPS) to interconnect devices in regards to the industry automation [14]. CPSs communicate with one another and with humans over an IoT infrastructure by "enabling intelligent industrial operation using advanced data analytics" $[3,15,16]$. Industrial IoT (IIoT) can be defined as the application of IoT on the industry.

An IoT solution is typically built as a three-layer architecture. First, a physical layer that includes sensors on end devices that collect data directly from the machinery. Then, there is a network layer that provides the infrastructure for remote communication with the physical layer. Finally, the application layer provides the ultimate functionality; this is the stage where the collected data are stored, processed, and analyzed [17,18].

A recent study shows the design of a vibration-based condition monitoring system in which the authors analyzed the design challenges for the required IoT architecture, prioritizing data volume. They also reviewed and compared multiple different potential technologies for the construction of end devices. When looking at the criteria they used for classifying the electronic components, the authors uncovered the importance of assuring a reliable performance for communicating large numbers of data. This assertion is accurate because of the quantity of raw data that a vibration analysis requires. Secondly, they intend to optimize the energy consumption, especially for portable end devices that are more likely to depend solely on the energy provided by the battery. Finally, the authors considered that obtaining a real-time response is important, since it allows the system to execute control in real-time. Therefore, transmission speed is also critical according to the authors [17].

The middle layer of IoT solutions for monitoring requires a Wireless Sensor Network (WSN) to communicate data from one host to another; ZigBee, Z-Wire, and 6loWPAN are some technologies available for this purpose [18]. Shahzad and $\mathrm{O}^{\prime}$ Nils deliberated over technologies such as WLAN, Bluetooth Low-Energy (BLE), and Zigbee; others were taken into account but discarded for experimentation. Furthermore, two types of hardware for on-device processing were also evaluated: microcontroller and FPGA. The experimentation 
they performed consisted of registering the devices energy consumption over different data throughput. The results showed how the energy consumption for WiFi is very consistent but not justifiable for throughput under $800 \mathrm{kB}$ [17].

Over the last decade, LoRa has been proposed as a potential solution to be implemented in short-range industrial applications. It has been considered even as a replacement for traditional technologies such as cellular networks and other short-range protocols commonly used [19]. Haxhibeqiri et al. note in their work that "LoRa is a candidate technology for low bandwidth industrial applications with a high number of communication devices spread across large areas" [20]. The low cost of the device and the low energy consumption are the main reasons multiple authors propose LoRa-based solutions for a variety of applications including IoT, smart homes, medicine, and industry, where they are exposed to rough environments both outdoor and indoors [20-22]. On their experiment, Haxhibeqiri et al. successfully simulated LoRa-based communication between two nodes separated up to 400 meters with a spreading factor of 12 . They also performed indoor experimentation involving multiple nodes supported a single gateway [20]. LoRa is a new world for network solutions for IoT, and many boards have been developed over the years, but Leonardi et al. gave special attention on their work to three different LoRa-based technologies because of their performance on noisy environments and potential Industrial applications. Those three variations of LoRa are LoRaWAN, Industrial LoRa, and RT LoRa features [19].

\section{Materials and Methods}

The overarching goal of this project is to design a remote sensing system in bearing housings for belt conveyor prognostics based on LoRa technology and over an industrial IoT architecture. In order to achieve this goal, we followed four strategic steps, where beforehand we established the application requirements and environmental conditions. Then, we drafted the possible solution to answer the requirements found on the previous belt conveyor characterization. Subsequently, the proposed solution structure led to the mechanic, electronic, and software design syntheses. The next stage was the manufacturing process of the designed devices. Finally, we verified the solution with a validation procedure, discussed in Section 4.

An initial study of the critical variables, operating conditions, and preventive maintenance activities of the target conveyor was carried out. It showed that the vibration and temperature of bearing housings are key variables for maintenance programming.

The maintenance department analyzes vibration empirically and only takes corrective actions when the failure condition is evident. The historical data of vibration and temperature records have also been intended for basic preventive maintenance. These data, together with the conveyor characterization and on field tests, described in Section 4, provided the initial parameters to guide the design process and instrument selection. The measurements data allow us to identify the range of the variables, expected peaks, and the required resolution.

Finally, given the environmental conditions around the target conveyor, the devices that are to be deployed on the field must be protected against dust and withstand exposure to rain. Therefore, we must consider a high Ingress Protection (IP [23]) on the mechanical design. On the other hand, the environmental conditions did not allow us to locate the processing data point (gateway) near the target transporter. This constraint brought the need to design a wireless network to reach remote device communication. Figure 1 shows that the gateway (b) is located about $150 \mathrm{~m}$ away from the belt conveyor (a) where the sensor modules are installed. 


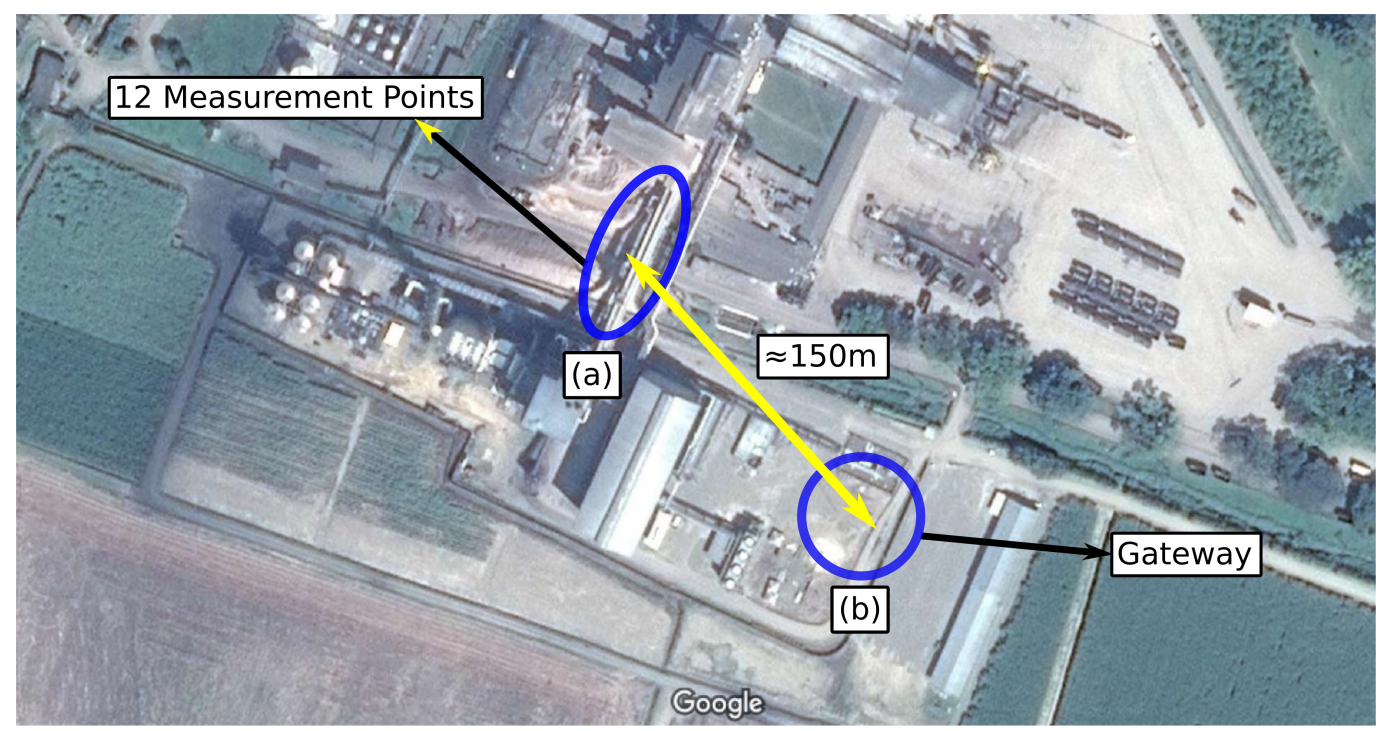

Figure 1. Geographic distribution of the communication points in the sugar cane mill under study. (a) Location of the belt conveyor of our study and the 12 measurement points of interest. (b) Location of the gateway. Source: Authors, Google Maps.

\subsection{Electronic Design Goals}

To carry out the electronic design, it was crucial to consider the behavior of several internal variables of the target system. Furthermore, some external variables such as environmental conditions should be considered since they affect both the production process and the measurements of the variables. We conducted an analysis of those variables and found that the vibration and temperature are critical components of failure analysis in our belt conveyor. Therefore, the system must take them both into account for performing failure predictions.

Our design requires the definition of the electronic components on each stage. First, the selection of an accelerometer and temperature sensor is discussed in Section 3.2. Subsequently, in Section 3.3, we describe the communication devices, focusing on the LoRa technology behind them.

On the other hand, one of the main challenges in the wireless communication system is the distance between the key points and the data acquisition gateway $(\approx 150 \mathrm{~m}$ on average; see Figure 1). The measurement nodes need to be located fixed at specific points in the conveyor's structure inside area (a) marked in Figure 1 and they will not be relocated in the future. The data acquisition gateway is to be located in area (b) (see Figure 1), where tools and equipment are available to connect the gateway to a Supervisory Control and Data Acquisition (SCADA) system.

The global proposed solution for the predictive maintenance system to be implemented in the mill under study is based on the IIoT strategy drawn in Figure 2, and it was designed as follows: in the first place, twelve bearing monitoring modules need to be located in the conveyor, composing the physical layer of the IoT architecture. Each monitoring module has a sensor in direct contact with the bearing housing. They are also connected to its corresponding signal conditioning and data acquisition system (DAQ). The transmission node embedded in the DAQ uses LoRa technology to open the link to the wireless network layer. This is located on the structure of the conveyor for major stability. The gateway completes the communication stage and establishes an interface to a computer with the SCADA application. This comprises the application layer. 




Figure 2. General conceptual scheme for predictive maintenance IoT Network in the sugar cane mill. The MP block represents the Motion Processing (MP) unit. Source: Authors.

\subsection{Sensors}

Our design requires a sensor profiling, which was carried out based on the range and sampling rate requirements of the overall system. We obtained these requirements from the technical report of the belt conveyor characterization. Subsequently, we focused on the bearing specifications and operational conditions. We must consider that the device will be exposed to a dusty environment and vibrations.

In the first place, it is required to measure axial, horizontal, and vertical acceleration in the main shaft of the reference bearing. The maintenance department does an assessment of velocity and the acceleration envelope to estimate the condition of the bearings. Given the above, it becomes crucial to analyze the measurements to obtain velocities and peak patterns. Historical data show that peaks of velocity can go between $0.31 \frac{\mathrm{mm}}{\mathrm{s}}$ and $11.48 \frac{\mathrm{mm}}{\mathrm{s}}$. For the acceleration envelope, they only measure the horizontal axis of the bearings, where the trend reaches $0.33 \mathrm{gE}$. Finally, acceleration values do not pass $1 \mathrm{~g}$ in optimal conditions. According to the evaluation from the vibration analyst in the sugar cane mill based on the severity chart of the ISO 1086 standard, vibration readings higher than $2 \mathrm{~g}$ - RMS trigger a fault alarm. However, some experts claim that the analysis of the intensity of the historical trend is more significant than just its instantaneous values [24,25].

The sampling rate plays also an important role in vibration analysis, since it depends on the rotational velocity of the bearings. The fastest shaft in the conveyor rotates at $99 \mathrm{rpm}$ $(1.65 \mathrm{~Hz})$; we consider this the operational frequency $\left(F_{o}\right)$. The Charlotte vibration chart is used to classify the type of fault according to the frequency spectrum [25]. This standard suggests that most of the faults are exposed between the first ten (10) multiples of $F_{o}$ on the frequency spectrum. When applying the Charlotte Standard in our project, as shown in Equation (1), the maximum frequency of the variable for the fastest shaft is calculated at $16.5 \mathrm{~Hz}$.

$$
\begin{aligned}
F_{o} & =1.65[\mathrm{~Hz}] \\
F_{\text {max }} & =F_{o} \cdot 10 \\
F_{\text {max }} & =16.5[\mathrm{~Hz}]
\end{aligned}
$$

The Nyquist-Shannon theorem states that the sampling frequency to reconstruct a signal should be at least two (2) times higher than $F_{\max }$. Yet, we should consider the 
unwritten rule of a multiplier factor $r>15$ instead of two to guarantee proper signal reconstruction [26]. Equation (2) shows the desired sample rate expression. As a result, an acceptable Sampling Frequency should be $F_{S}>248[\mathrm{~Hz}]$. Thus, all faults described in the Charlotte vibration chart shall be detected.

$$
F_{s}=r \cdot F_{\text {max }}
$$

Vibration analysis is carried out using acceleration measurements. In this study, we assessed multiple accelerometer sensors, and the most relevant ones are listed in Table 1.

Table 1. Accelerometer sensors specifications.

\begin{tabular}{cccccc}
\hline Sensor Device & Number of Axes & Acceleration Range & Sampling Frequency & Resolution/ Sensitivity & Price \\
\hline PCE-AC500G & 1 & $\pm 500 \mathrm{~g}$ & $0.65 \ldots 23,000 \mathrm{~Hz}$ & $10 \mathrm{mV} / \mathrm{g}$ & $344.66 \mathrm{USD}$ \\
\hline CISS Bosh & 3 & $\pm 16 \mathrm{~g}$ & $\pm 100 \mathrm{~Hz}$ & $14 \mathrm{bit}$ & $454.80 \mathrm{USD}$ \\
\hline ADXL345 & 3 & $\pm 16 \mathrm{~g}$ & 6.25 to $3200 \mathrm{~Hz}$ & $13 \mathrm{bit}$ & $25.33 \mathrm{USD}$ \\
\hline MPU 9250 & 3 & $\pm 16 \mathrm{~g}$ & $1000 \mathrm{~Hz}$ & $16 \mathrm{bit}$ & $13.68 \mathrm{USD}$ \\
\hline
\end{tabular}

The PCE-AC500G has a wide operational range of $\pm 500 \mathrm{~g}$, and it has the highest sampling rate out of the four sensors; this sensor is especially used in spacecraft applications such as rockets. The Bosch CISS is a smart sensor with a smaller but sufficient operational range and integrates USB communications. However, this sensor provides the lowest sampling frequency, which does not meet the system requirements. The ADXL354 is a low-cost accelerometer with a full-scale range of $\pm 16 \mathrm{~g}$ and $\mathrm{I} 2 \mathrm{C}$ communication hardware integrated. On the other hand, the MPU9250 has similar characteristics to the ADXL354 but with lower sampling rate. Even so, a $1 \mathrm{kHz}$ samplig rate is still acceptable for the present application. Given that almost all sensors fulfill the desired specifications, the sensor price conditioned the final decision. Therefore, we chose the commercial MPU9250. Figure 3 shows the schematic and board of the sensor.

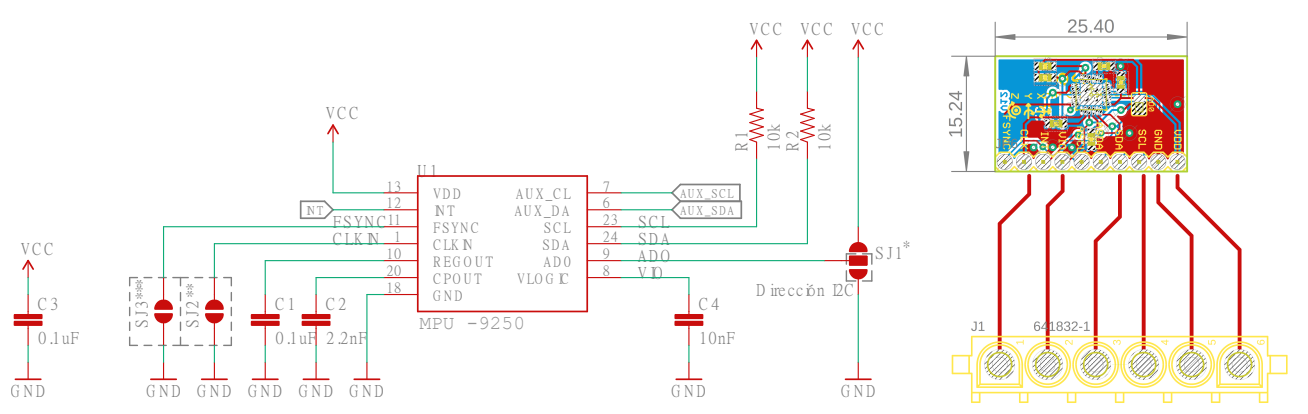

Figure 3. Electrical scheme of sensor module MPU9250. Source: Adafruit Industries.

From historical reports, the mean temperature was taken to be $31.32{ }^{\circ} \mathrm{C}$. The lowest reported measure was $24{ }^{\circ} \mathrm{C}$ on night shifts, while the expected maximum temperature is $40{ }^{\circ} \mathrm{C}$. The maintenance department considers a possible fault when the temperature value goes higher than $40{ }^{\circ} \mathrm{C}$. For the selection of the temperature sensor, we considered instruments that provided at least $80{ }^{\circ} \mathrm{C}$ of maximum temperature. The MLX90614 and AMG8833 are non-contact sensors that comply with the temperature range requirements; however, they are not ideal for an industrial application with a lot of environmental dust. The belt transportation system for bagasse on the sugar cane mill is very susceptible to spilling its content which accumulates on the bearing housing surface where tick layers are formed. Because of this, all contact temperature sensors are adequate for the environment of our project. Contact sensors similar to the SHT10 are more suitable; nevertheless, the MPU9250 has an embedded temperature sensor with a wide enough range from $-40{ }^{\circ} \mathrm{C}$ to $85^{\circ} \mathrm{C}$ and a digital output signal. 


\subsection{Transmission Device}

The network layer of our application needs thirteen transmission nodes, one for each monitoring module, and an extra one for the gateway. We designed the twelve transmission devices to be located close to each bearing, while the gateway location was selected looking to guarantee the best signal strength and attempting to reduce communication interference. In accordance with the internal business restrictions, the best possible location for the gateway was $150 \mathrm{~m}$ away from the measurement points, as shown in Figure 1.

To select the appropriate technology, four of our applications we evaluated the following factors: operation range, price, energy consumption, and outdoor experimentation performed. The data rate was not a decisive factor because measurements are first collected in place and then sent with a small delay.

Both the measurement nodes and the gateways use a LoRa32U4 device as the main component. Furthermore, the on-device transmission module uses a Real-Time Clock (RTC) (DS3231) and an Electrically Erasable Programmable Read-Only Memory (EEPROM) as complementary elements that interconnect through an Inter-Circuit Interface Communication (I2C) protocol. Figure 4 shows the internal structure of both the gateway and measurement nodes.

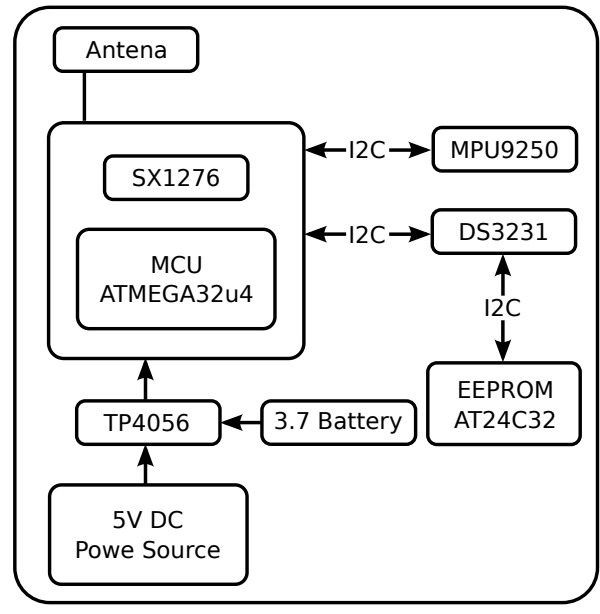

(a)

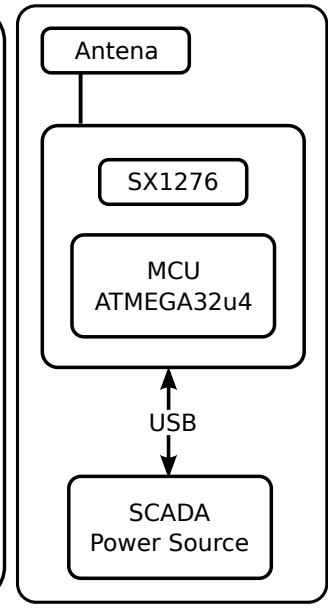

(b)

Figure 4. Block diagram of the network layer components: (a) Structure of measurement node. (b) Structure of gateway. Source: Authors.

Figure 4 shows the internal communication structure of the acquisition/transmission (a) and reception (b) modules. The vibration and temperature sensor (MPU9250) takes the data and communicates them to the processing block and transmission (ATMEGA32u 4 and SX1276). In (a), there is also a battery management system (TP4056) for when the module loses external power, while the Real-Time clock (RTC) module (DS3231) is used to keep a timestamp of each measurement. In (b), internal components of the reception module, also gateway, are displayed; this Microcontroller Unit (MCU) is the master of the LoRa communication and coordinates the reception of the data and its subsequent transfer to the SCADA.

The LPWAN connection topology is a direct connection between the nodes, and there are no intermediate systems or modules called single-hop stars. The end devices are connected directly to the base station via media access control (MAC) protocols. This type of connection between the elements of the system simplifies the network, gives it robustness, and generates centralized control [27]. Due to the characteristics of simplicity and robustness, it is chosen to be applied in the project. 
LoRa

LoRa technology is an example of an LPWAN system communication. It is similar to wireless data transmission networks such as WiFi, Bluetooth, LTE, or SigFox. In its beginning, LoRa was an initiative of a group of enterprises such as Semtech, IBM, and Actility, among others. They make up the LoRa Alliance. This technology uses a proprietary radiofrequency modulation technique patented by the LoRA Alliance [28].

LoRa-based communication networks have been shown to be highly suitable for IoT applications, where devices are generally unplugged from the network power supply. Recent works such as $[19,29,30]$ show the applicability of LoRa technology in an industrial environment, as mentioned in Section 3.3. To establish an IoT connection using LoRa technology, we must create a Wireless Personal Area Network (WPAN). Thus, it was needed to develop our gateway and node devices. Fortunately, it is possible to adapt the communication network to the needs of a particular application.

The LoRa 32u4 has an ATMega32u4 microcontroller (MCU) in charge of the communication protocol and data management. This MCU is interconnected to a SX1276 that enables the LORA wireless communication; the SX1276 module uses a Radio Frequency Modulation (RFM) set up at $915 \mathrm{MHz}$ that is represented in the schematic shown in Figure 5.

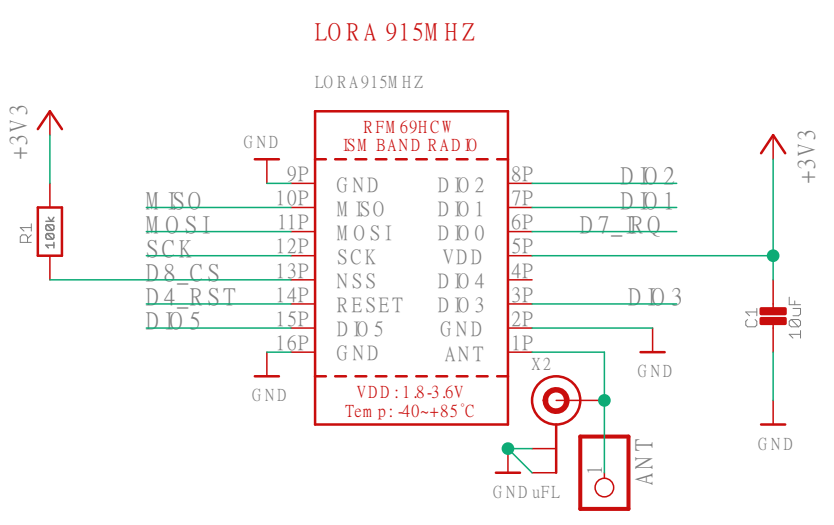

Figure 5. Electrical scheme of LoRa 32u4 II module. Source: Adafruit Industries.

The data transmission module must be able to send the information fast enough to be processed by the SCADA software and have the power so the elements that can generate interference do not deteriorate the condition of the transmitted data. For the application within the system, the LoRa device is chosen with the following features:

- Dimensions $51 \mathrm{~mm} \times 23 \mathrm{~mm} \times 8 \mathrm{~mm}$

- Weight $5.5 \mathrm{~g}$

- Processor ATmega32u4-8 MHz

- $\quad$ Number of Pins 20 GPIO

- $\quad$ Number of PWM Pins 7

- Number of Analog Inputs 10

- Hardware Support I2C

- $\quad$ Radio Module $868 / 915 \mathrm{MHz}$

Figure 4 shows the architecture of the connections of the monitoring and transmission module. There is a voltage regulator as a safety element for the components that make up the described system. In both there is an ATMega32u4 microcontroller that is responsible for the reception and management within the transmission module of the data obtained by the sensor placed on the bearing.

On the transmission node, the ATMega32u4 coordinates tasks of reading data from the sensor upon receiving an order from the gateway. It also coordinates the communication protocol to send the collected data to the gateway. Figure 6 shows the flowchart of the algorithm on the monitoring module, identifying the three decision sequences that the module can take: 1 , Send service information about the module such as ID, name of bearing, 
and sampling parameters; 2, Measure temperature value for alarm detection; 3, Enable acceleration sampling sequence.

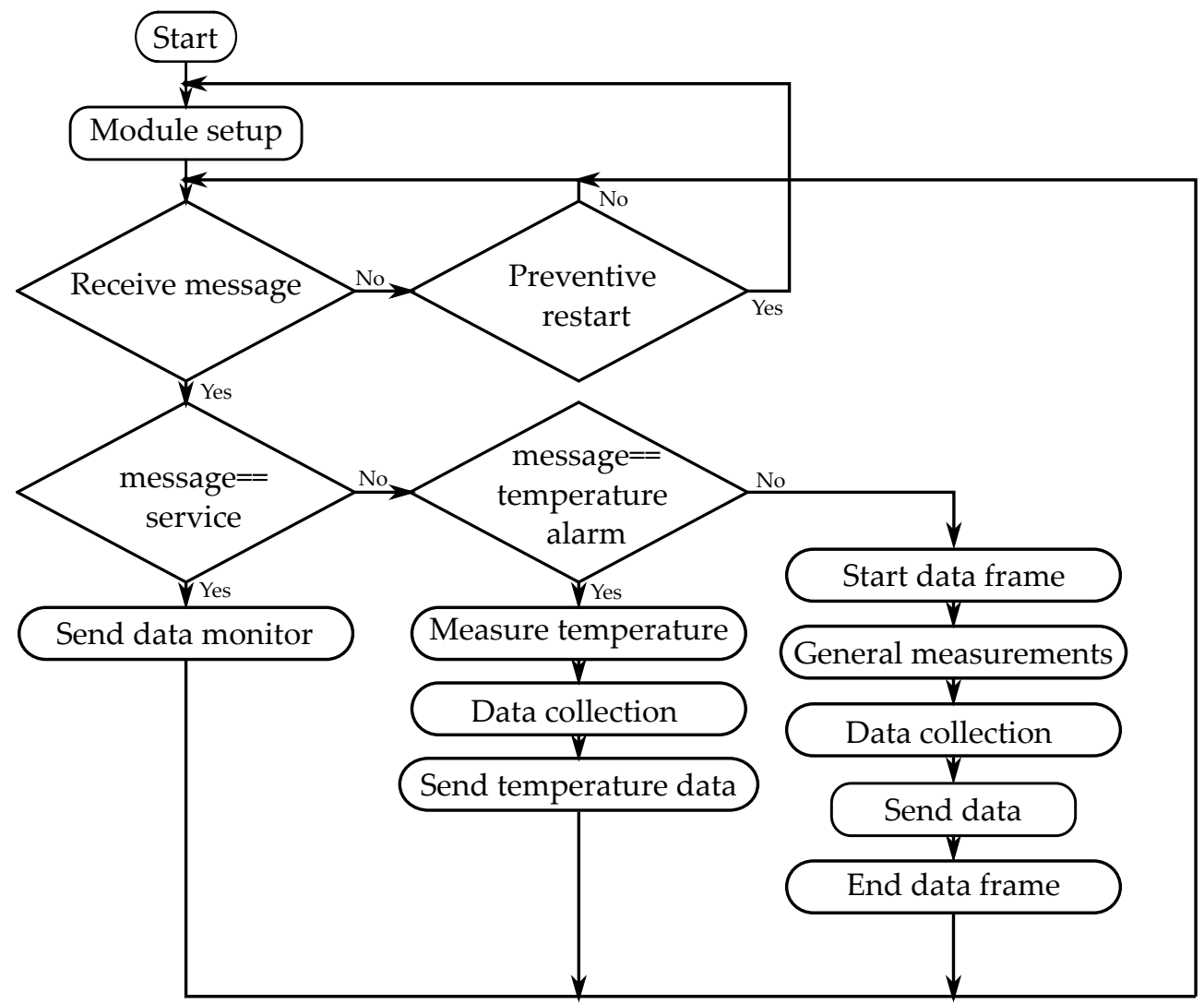

Figure 6. Block diagram of sensing and communication routines. Source: Authors.

Internal communication between modules is based on a command-response protocol. It is governed by the reception module, which is assigned as the master and is identified with the ID 00. Each transmission module is a slave identified with a unique ID. The firmware was programmed so that every request sent by the master is repeated up to four times. If a transmission module where the data are collected does not respond, a communication alert is generated that goes directly to the SCADA.

The reception module can be set up either on manual or automatic mode. This setting is sent through the serial port as well as the codes of the commands when operating on manual. When configured on automatic, the receiver first requests for the state of the temperature alarms to each transmission module and then asks for the acceleration data of the first slave. Then the master asks again for all the alarms status before continuing with the second slave; this sequence is repeated indefinitely.

In the data structure for the acceleration, the measures are quantified. This number is sent within a frame indicator inside the package that contains data of three axes. When the master receives an acceleration package, the frame indicator is compared internally. If the indicator is confirmed, the master sends a validation code to the slave. With the confirmation from the master, the slave continues the transmission of the next package. Otherwise, an error message is sent so the slave repeats the transmission of the last package.This cycle can be repeated up to four times; if the error continues the communication is considered as lost, and an alarm is triggered.

\subsection{Assembly of Printed Circuit Board}

Upon validation of the remote sensing system design, including a first data acquisition and laboratory test with a prototype, we proceeded with the manufacture of the PCBs. Figure 7 shows from left to right the design of the PCB on two layers differentiated with 
blue and red colors. Then, it is followed by a photo of each of the face of the actual printed board.
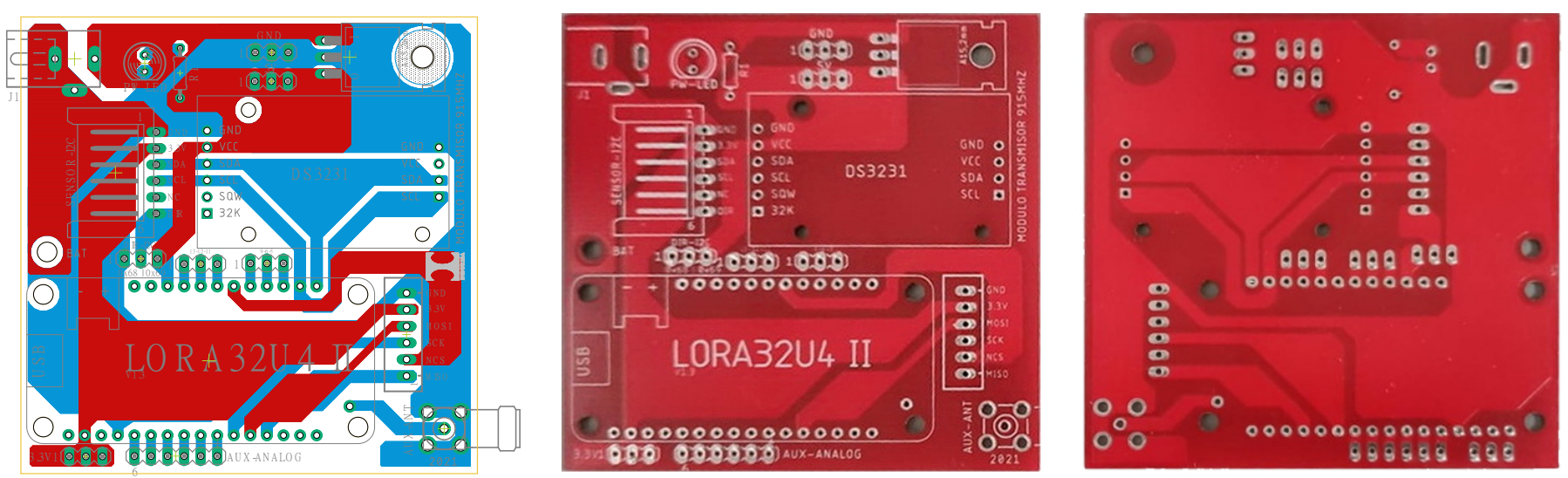

Figure 7. Printed Circuit Board (PCB). Designed PCB, face 1 and 2 of built PCB. Dimensions $70 \mathrm{~mm} \times 70 \mathrm{~mm}$. Source: Authors.

\subsection{Mechanical Design}

The mechanical design was carried out considering the size of the bearings and the way in which they must be placed in order to prevent affecting the operation of the system. The size of electronic components also affects the mechanical design of measurement devices. The measurements of the box that protects the electronic devices must be kept in proportion to properly fit the bearings. It is also important to maintain protection against the ingress of water and dust, as these can affect both the measurements and data transmission. The mechanical design includes IP protection, which guarantees that the system is not affected by external particles that could damage the device. Section 4.2 provides a more detailed way to design IP protection.

Figure 8 shows the mechanical design of the compartment that protects the sensor module MPU9250 located over the bearing housing. The wired connection of the sensor to the data collection module shown in Figure 9 is protected by a cable gland.
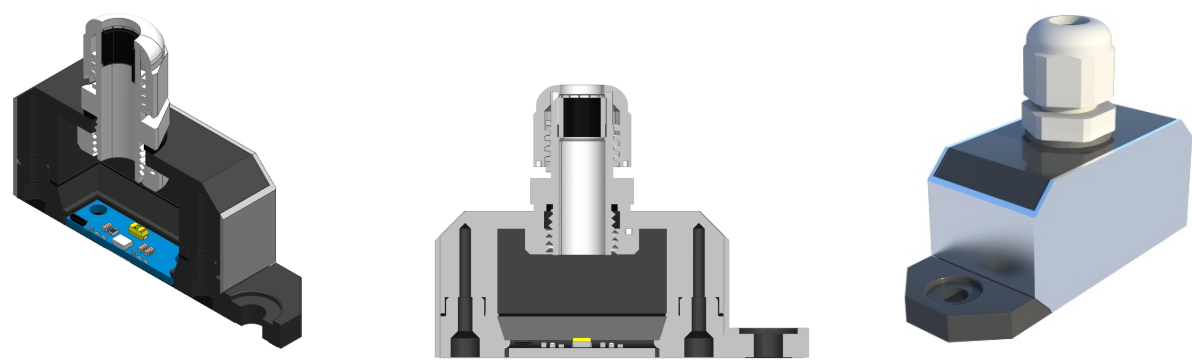

Figure 8. Computer-Aided Design (CAD) model for electronic and case of the measurement modules. Source: Authors.

Every measurement node located in the conveyor must have at least an IP66 protection. This means that dust particles under $50 \mu \mathrm{m}$ (reference [31] defines the particle dimensions for the corresponding protection factors) and any directional thrown water should not ingress the devices [32]. 
The transmission modules must take into account the same IP characteristics as the data collection modules. The assembly of the communication modules must not affect the conveyor operation. Furthermore, the internal electronic components must be protected from the entry of foreign elements in the form of particles or the entry of water, which would be the worst case scenario. An IP66 protection level was also selected for the transmission modules. Figure 9 shows the CAD appearance of the communication modules.

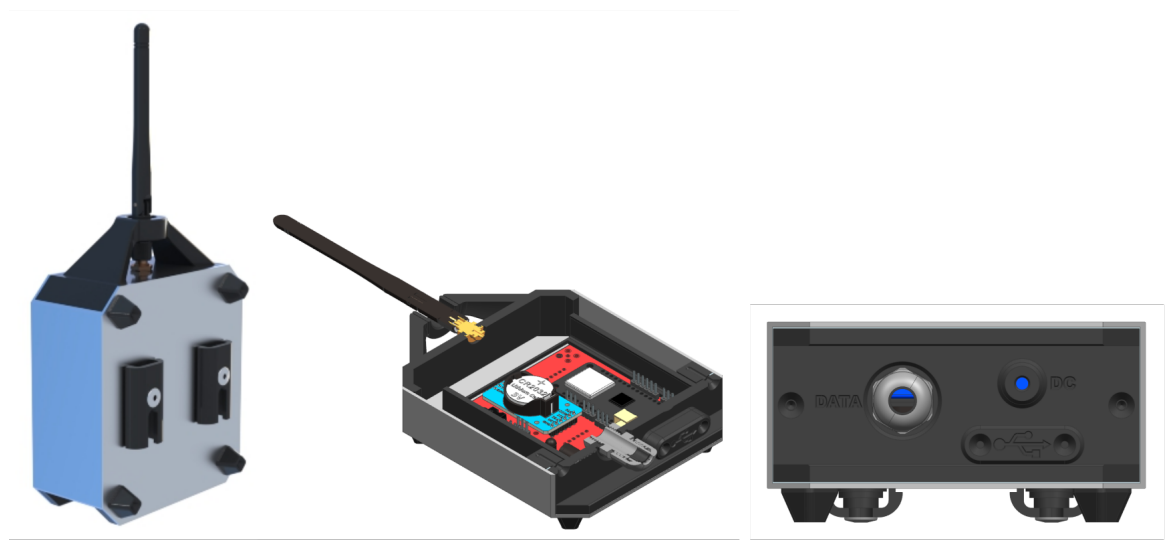

Figure 9. CAD model for electronic plate and case of the transmission modules. Source: Authors.

\section{Experiments and Results}

In order to guarantee the reliability of the designing process described, and make an assessment of the devices, we executed several different laboratory experiments, simulations, and field tests.

This section describes the experiments performed to aid the reliability of the manufacturing process of the remote sensing system. Some of these experiments are thermographic analysis, IP simulation, and vibration test. Furthermore, some operational test were performed in order to make an assessment of the operation and reliability of the sensing and communication devices.

\subsection{Thermographic Analysis}

For preventive and predictive maintenance, temperature supervision is critical for fault detection in machines. On belt conveyors, the temperature monitoring focuses on the bearing and gearmotor housings because they contain most rotating parts such as rings, rollers, bushings, and shafts. The heat on bearing housings is generated from the friction between its moving parts. Most of these parts are not reachable from the outside of the housing, although heat flows towards the housing surface. However, we cannot assume a uniform heat distribution. Then, a thermographic analysis with a Fluke camera Ti200-16110220 was carried out.

Figure 10 shows an example of the thermographic analysis. It revealed that the highest temperatures are concentrated around the shaft, where the average temperature on most bearings is $37^{\circ} \mathrm{C} \pm 2{ }^{\circ} \mathrm{C}$, while the housing average temperature on all bearings is $29^{\circ} \mathrm{C}$ $\left(\mathrm{s}=1.9^{\circ} \mathrm{C}\right)$. On the other hand, the greatest gap in temperature was found on the bearing close to the engine transmission, where the measurement was $56^{\circ} \mathrm{C}$ around the shaft. Over the thermal image of each bearing, an area was selected for a deeper analysis. Those areas did not exhibit significant temperature drifts. Furthermore, the zone with the greatest variations has a standard deviation of $0.52{ }^{\circ} \mathrm{C}$. 


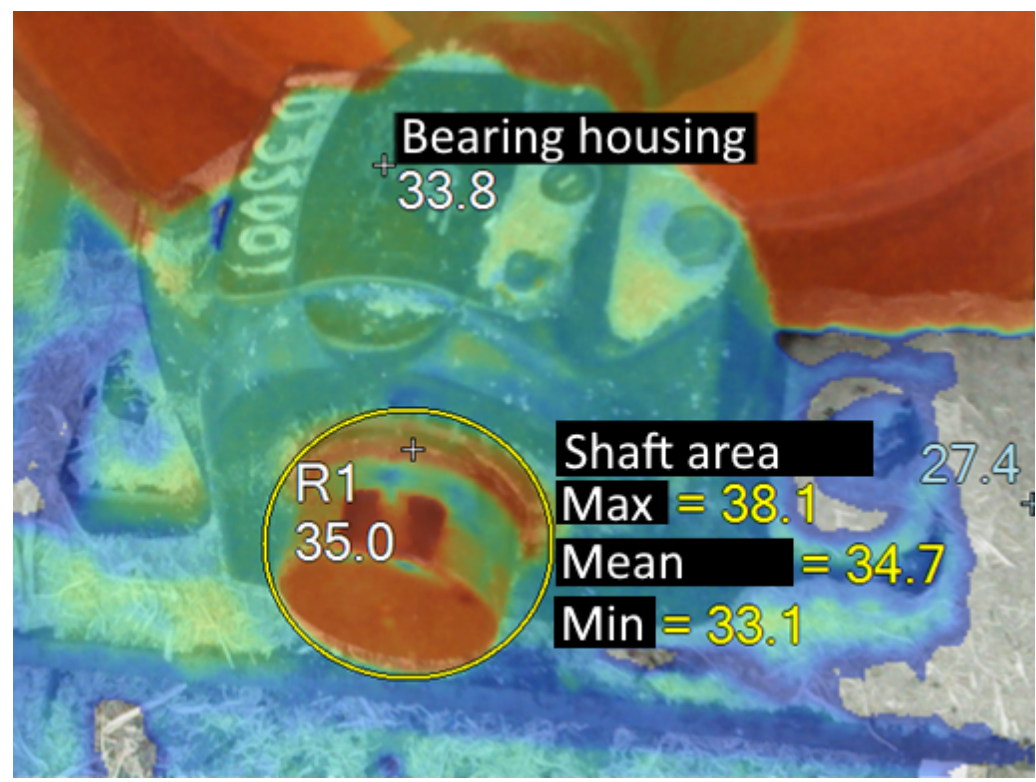

Figure 10. Thermographic image of the bearing housing. Source: Authors.

\subsection{IP Simulation}

One of the main attributes that our devices must have is ingress protection. From the conveyor characterization, we decided that the system must have at least an IP66 protection level (see [23] for details on IP concepts). We performed a Computational Fluid Dynamics (CFD) simulation to reproduce an assessment of the IP level required. We use the Ansys Fluent software, where we imported a simplified 3D model from a Computer Aided Design (CAD) software.

The CFD simulated a high-pressure jet according to water protection level 6. During the evaluation, the water jet was launched vertically as well as horizontally, as shown in Figure 11. The test allowed us to validate the ingress protection for the devices to operate on the conveyor at the sugar cane mill.

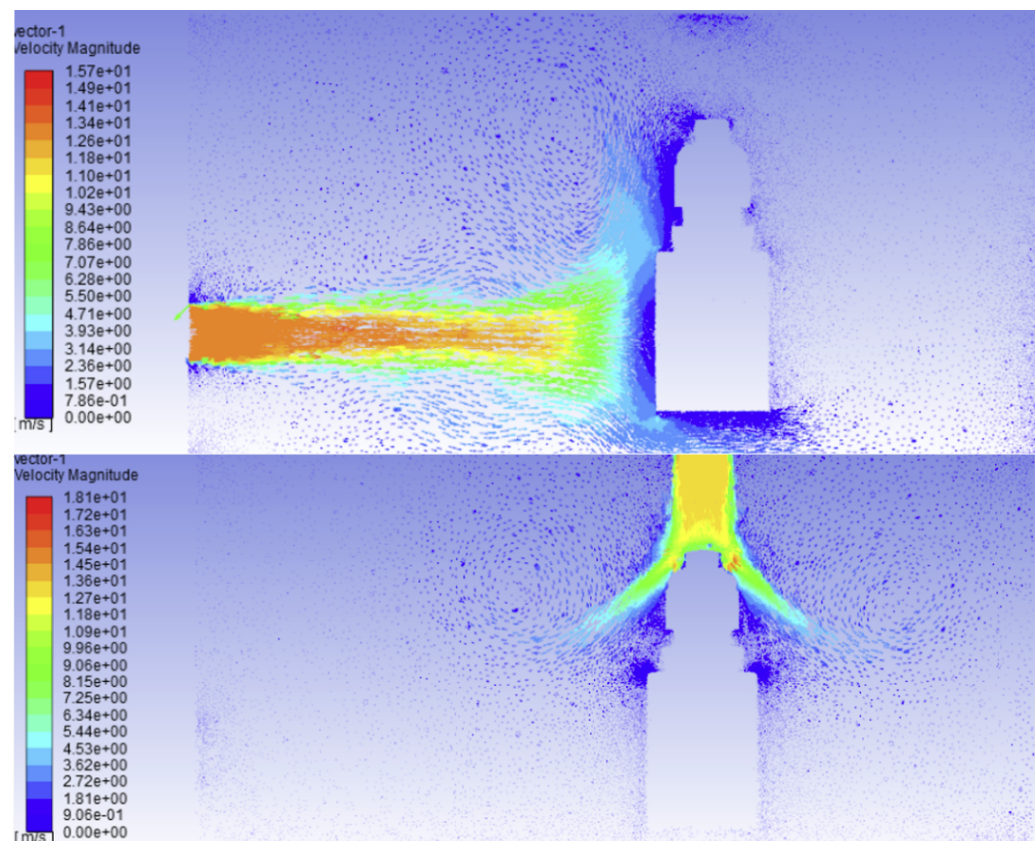

Figure 11. Computational fluid dynamics simulation of the sensor on Ansys Fluent software, the CAD model of the sensor is shown in Figure 8. Source: Authors. 


\subsection{Mechanical Stability Assessment through Vibration Test}

The purpose of any vibration test is to establish material qualities and uncover design and manufacturing faults. This evaluation intends to guarantee the compatibility of a developed device with its future usage environment.

For this experiment, we completed two individual procedures for each of the three axes (vertical, horizontal, and axial) of the manufactured monitoring module. In the first place, we carried out an operational test to verify the worst case scenario. The hypothetical situation simulated the roughest environmental vibrations the devices can undergo amid operation. Secondly, a resistance test reveals failures related to the long-time usage.

Before, during, and after the vibration test, the mechanical and electrical integrity of the device was evaluated. Throughout the experiment, we also monitored the temperature with a Fluke thermography camera Ti200, of which Figure 12 shows a photo. As the experimentation progressed and the vibration generated from the shaker were augmented, the device temperature increased accordingly. This behavior was expected, and the recorded values never endangered the equipment. The most important conclusion of the vibration test is that the electronic elements did not suffer any damage, and the PCB kept its electrical properties. Therefore, the monitoring module is adequate for operating on the belt conveyor of the sugar cane mill.


Figure 12. Mechanical and stability test on vibration test rig. Source: Authors.

\subsection{Operation Tests}

Operational tests are intended to determine if the measurements are taken correctly and if the information is sent correctly. It is essential to estimate the expected error in the measures to define the reliability of the devices in terms of accuracy. Similarly, it is important to determine the reliability of the transmission devices in terms of the completeness and correctness of the sent information. Section 2 brings to relation several works where data-driven approaches for machinery prognostics have been used in similar applications.

Some fundamental studies in data analytics, specifically in deep learning models and algorithms, show the relevance of uncertainty awareness in supervised learning frameworks. For example, Kendall et al. in [33] offer an extensive review of the required uncertainty considerations for data-driven estimators. Although the review is focused on computer vision applications, relevance and basic concepts about required uncertainties hold. More recently, uncertainty-aware methodologies for prognostics have been presented, for example, in [34-36]. They show that uncertainties put conditions over the behavior of the estimators. Furthermore, they show that the uncertainty level of the input information puts a boundary on the final expected performance of the prediction models. This makes it important to estimate our devices' errors and uncertainty levels to define the expected reliability in our future applications.

We have carried out a statistical experiment to assess the operational reliability of the entire sensing network and communications devices. This experiment allows us to specify the reliability of the devices in terms of two aspects: (i) the deviations from the actual values of the measured variables and (ii) the robustness of the communication devices. To 
determine the size of the experiment, we took into account the operational conditions of the devices. We estimate the number of independent runs in our study for each test using the central limit theorem from the approach described in [37] and more recently in [38]. Specifically, we need to highlight some specific definitions:

- Population Size N: It refers to the number of single subjects in the study. In the case of communications, it is the number of data packets sent to the gateway. In the case of measurements, it is the number of temperature and vibration time windows recorded.

- Confidence Interval $c$ : This is the desired level of confidence in the obtained results from the statistical experiment. In other words, this is the probability of the conclusion being correct. Using this confidence interval, the statistic $Z_{c}$ is obtained from an inverse Gaussian Cumulate Density Function (CDF).

- Sample Proportion $p$ : This is the estimated ratio between correct cases and the total number of cases.

- Estimation Error $e$ : This is the confidence interval around the sample proportion. In other words, the resulting sample size will guarantee results with sample proportion errors less than $e$.

Table 2 shows the chosen parameters for the test. The infinite population size $N$ means that we do not place bounds on the lifecycle of our device. The desired confidence interval is $99 \%(c=0.99)$, with an estimation error less than $5 \%(e=0.05)$; we choose the ratio of wrong cases to be, in terms of sample size, the worst case $(p=0.5)$. The sample size can be computed as shown in Equation (3) [37].

$$
M=\frac{Z_{c} \cdot p(1-p)}{e^{2}}
$$

From Equation (3), we can see that the communication and data acquisition tests need at least $664(M=663.489)$ samples.

Table 2. Parameter set for operational testing.

\begin{tabular}{ccl}
\hline Parameter & Value & \multicolumn{1}{c}{ Description } \\
\hline$N$ & $\infty$ & Population size \\
\hline$p$ & 0.5 & Worst case of right-wrong cases rate \\
\hline$c$ & 0.99 & Level of confidence \\
\hline$e$ & 0.05 & Maximum estimation error \\
\hline$Z_{c}$ & 2.58 & Statistic for confidence interval from a normal Gaussian distribution \\
\hline
\end{tabular}

The operation tests were executed in the real environment, where the modules were arranged as described in Figure 1. The climatic conditions were considered normal, with a temperature of $24^{\circ} \pm 6^{\circ}$, and minimal to no rainfall.

\subsubsection{Communication Test}

In the communication test, each device needs to perform the correct pairing (transmissionreceiving) of at least 664 messages in a row. If the gateway correctly receives the whole amount of messages, we can conclude with $99 \%$ confidence that the communication device will operate properly during its life cycle. Table 3 summarizes the results of these experiments. 
Table 3. Data Acquisition and Communication tests.

\begin{tabular}{ccccccc}
\hline \multirow{2}{*}{ Node } & \multicolumn{2}{c}{ Transmission } & \multicolumn{4}{c}{ Measurements RMRSE } \\
\cline { 2 - 7 } & Sent & Correct & M & RMS & PEAK & TEMP \\
\hline 1 & 670 & 670 & 670 & 0.00115 & 0.00960 & 0.00382 \\
\hline 2 & 670 & 670 & 670 & 0.00113 & 0.00878 & 0.00392 \\
\hline 3 & 670 & 670 & 670 & 0.00103 & 0.00715 & 0.00401 \\
\hline 4 & 670 & 670 & 670 & 0.00099 & 0.00794 & 0.00426 \\
\hline 5 & 670 & 670 & 670 & 0.00117 & 0.00795 & 0.00381 \\
\hline 6 & 670 & 670 & 670 & 0.00094 & 0.00643 & 0.00436 \\
\hline 7 & 670 & 670 & 670 & 0.00105 & 0.00867 & 0.00417 \\
\hline 8 & 670 & 670 & 670 & 0.00102 & 0.00793 & 0.00397 \\
\hline 9 & 670 & 670 & 670 & 0.00098 & 0.00753 & 0.00410 \\
\hline 10 & 670 & 670 & 670 & 0.00076 & 0.00888 & 0.00399 \\
\hline 11 & 670 & 670 & 670 & 0.00107 & 0.00838 & 0.00412 \\
\hline 12 & 670 & 670 & 670 & 0.00082 & 0.00910 & 0.00435 \\
\hline & Average & & 0.00101 & 0.00820 & 0.00407 \\
\hline
\end{tabular}

\subsubsection{Data Acquisition Test}

The data acquisition test is an assessment of the measurement devices. We carried out the measurement of 670 samples and evaluated the relative mean root square error (RMRSE; see Equation (4)) for the root mean square (RMS) acceleration, peak acceleration, and instantaneous temperature.

$$
\text { RMRSE }=\sqrt{\frac{1}{M} \sum_{i=1}^{M}\left(\frac{d_{i}-\hat{d}_{i}}{d_{i}}\right)^{2}}
$$

where $M$ is the number of measurements $(M=670), \hat{d}_{i}$ is the data value measured by our device, and $d_{i}$ are reference measurement values obtained from an IR sensor and the probe CMSS 797 for the acceleration. Both sensors are used in our company for preventive maintenance activities. Table 3 shows a summary of results. The statistical experiment allows us to specify the mean and deviation values with $99 \%$ probability that they are accurate. These results are relevant to perform prognostics and predictive maintenance in future works. The quality and uncertainty of predictions provided by state-of-the-art data analytic methods and models are highly dependent on the quality of self-data $[10,25]$.

\section{Discussion}

The thermographic analysis was a key component of the conveyor characterization. This, together with historical temperature and vibration registers, led to decisions being made through the design phases in regards of the operating conditions and measurement ranges. Specifically, termographic images were useful to define the measurement points on the bearing housing surface. From Figure 10 and other thermic images, we obtinaed an operation range between environmental temperature and $50{ }^{\circ} \mathrm{C}$ in the worst fault cases. This analysis supported the process for selecting the temperature sensor and ratify the data provided by the factory.

Regarding the IP test simulation, it allowed us to validate the mechanical design process. Figure 11 in Section 4.2 shows an screenshot of the CDF simulation that probed the device protection to water and particles above $50 \mu \mathrm{m}$ from lateral and upper sources. This result leave us to give the IP66 feature to the designed physical device. 
Finally, the electronic devices for measurement and communications were tested under continuous operating conditions. Table 3 shows that all the communication pairings (transmitting-receiving) in the test were correct. That means they will have a reliability of $99 \%$ during their normal operation, as stated in the statistical experiment. Furthermore, in the measurement test, the table shows the following mean relative errors:

- Under $0.12 \%$ for the RMS acceleration

- Under $1.00 \%$ for peak acceleration

- Under $0.50 \%$ for temperature.

Therefore, based on the statistical experiment parameters, we can say that, with a confidence interval of above $99 \%$, our device has the following measurement specifications:

- $\quad$ Acceleration measurement: $a \pm 0.0012 a$

- $\quad$ Temperature measurement: $T \pm 0.0005 T$

These features are adequate and relevant for condition estimation and predictive maintenance. For future work in the short and middle term, we are willing to collect a vibration and temperature database, which in the medium and long terms are going to be used to train learning models intended for machinery condition estimation and subsequent fault predictions. Alongside the local database, further operation tests must be done to validate the reliability of the system in the long term. This work is a step toward bringing the technology of industry 4.0 to our local companies.

Author Contributions: Conceptualization, M.C. (Mariana Cardona), B.H., and W.P.; methodology, B.H., M.C. (Michael Cifuentes), and W.P.; software, B.H. and M.C. (Michael Cifuentes); validation, M.C. (Mariana Cardona) B.H., and W.P.; formal analysis, B.H.; investigation, M.C. (Mariana Cardona) and B.H.; resources, M.C. (Mariana Cardona), M.C. (Michael Cifuentes), and W.P.; data curation, M.C. (Mariana Cardona) and W.P.; writing—original draft preparation, M.C. (Mariana Cardona) and M.C. (Michael Cifuentes); writing-review and editing, B.H. and W.P.; visualization, M.C. (Michael Cifuentes) and M.C. (Mariana Cardona); supervision, W.P.; project administration, B.H. and W.P.; funding acquisition, W.P. All authors have read and agreed to the published version of the manuscript.

Funding: This research was funded by Ministerio de Ciencias Tecnología e Innovación (MinCiencias), Servicio Nacional de Aprendizaje (SENA), and Industrias del Risaralda S.A.S. (INDURISA) grant “Programa Línea de Fomento a la Innovación y Desarrollo Tecnológico en las Empresas, Convocatoria 851-2019".

Institutional Review Board Statement: Not applicable.

Informed Consent Statement: Not applicable.

Data Availability Statement: Publicly available datasets were analyzed in this study. This data can be found here: [https://github.com/ByronHernandez/LPWSN] (accessed on 15 November 2021).

Conflicts of Interest: The authors declare no conflict of interest.

\section{References}

1. Motaghare, O.; Pillai, A.S.; Ramachandran, K.I. Predictive Maintenance Architecture. In Proceedings of the 2018 IEEE International Conference on Computational Intelligence and Computing Research (ICCIC), Madurai, India, 13-15 December 2018; pp. 1-4. [CrossRef]

2. Cheng, C.; Zhang, B.; Gao, D. A Predictive Maintenance Solution for Bearing Production Line Based on Edge-Cloud Cooperation. In Proceedings of the 2019 Chinese Automation Congress (CAC), Hangzhou, China, 22-24 November 2019; pp. 5885-5889. [CrossRef]

3. Lodewijks, G.; Li, W.; Pang, Y.; Jiang, X. An Application of the IoT in Belt Conveyor Systems. In Internet and Distributed Computing Systems; Li, W., Ali, S., Lodewijks, G., Fortino, G., Di Fatta, G., Yin, Z., Pathan, M., Guerrieri, A., Wang, Q., Eds.; Springer: Cham, Switzerland, 2016; pp. 340-351.

4. Blazej, R.; Jurdziak, L.; Kirjanow-Blazej, A.; Kozlowski, T. Identification of damage development in the core of steel cord belts with the diagnostic system. Sci. Rep. 2021, 11, 12349. [CrossRef] [PubMed]

5. Skoczylas, A.; Stefaniak, P.; Anufriiev, S.; Jachnik, B. Belt Conveyors Rollers Diagnostics Based on Acoustic Signal Collected Using Autonomous Legged Inspection Robot. Appl. Sci. 2021, 11, 2299. [CrossRef] 
6. Lodewijks, G. Strategies for Automated Maintenance of Belt Conveyor Systems. Bulk Solids Handl. 2004, 24 , 16-22.

7. Stefaniak, P.; Wodecki, J.; Zimroz, R. Maintenance Management of Mining Belt Conveyor System Based on Data Fusion and Advanced Analytics. In Advances in Technical Diagnostics; Timofiejczuk, A., Łazarz, B.E., Chaari, F., Burdzik, R., Eds.; Springer: Cham, Switzerland, 2018; pp. 465-476.

8. Zimroz, R.; Hardygóra, M.; Blazej, R. Maintenance of Belt Conveyor Systems in Poland-An Overview. Available online: http: / / diagbelt.pwr.edu.pl/Publikacje/Maintenance\%20of\%20Belt\%20Conveyor\%20Systems.pdf (accessed on 15 November 2021).

9. Stefaniak, P.K.; Wyłomańska, A.; Obuchowski, J.; Zimroz, R. Procedures for Decision Thresholds Finding in Maintenance Management of Belt Conveyor System-Statistical Modeling of Diagnostic Data. Available online: https://www.researchgate.net/profile/ Pawel-Stefaniak/publication/266088270_Procedures_for_decision_thresholds_finding_in_maintenance_management_of_ belt_conveyor_system_-_statistical_modeling_of_diagnostic_data/links/5425425a0cf26120b7ac8677/Procedures-for-decisionthresholds-finding-in-maintenance-management-of-belt-conveyor-system-statistical-modeling-of-diagnostic-data.pdf (accessed on 15 November 2021).

10. Baptista, J. Industrial Maintenance: Techniques, Stores, and Cases; CRC Press: Boca Raton, FL, USA, 2020.

11. D'Angelo, T.; Mendes, M.; Keller, B.; Ferreira, R.; Delabrida, S.; Rabelo, R.; Azpurua, H.; Bianchi, A. Deep Learning-Based Object Detection for Digital Inspection in the Mining Industry. In Proceedings of the 2019 18th IEEE International Conference On Machine Learning and Applications (ICMLA), Boca Raton, FL, USA, 16-19 December 2019; pp. 633-640. [CrossRef]

12. Nienhaus, K.; Warcholik, M.; Büschgens, C.; Müller, D. Belt Positioning and Skewing Prevention in Lignite Mining Using Long-Wavelength Infrared Cameras. In Proceedings of the 12th International Symposium Continuous Surface Mining-Aachen 2014; Niemann-Delius, C., Ed.; Springer: Cham, Switzerland, 2015; pp. 11-20. [CrossRef]

13. Mazurkiewicz, D. Computer-aided maintenance and reliability management systems for conveyor belts. Eksploat. Niezawodn. 2014, 16, 377-382.

14. Boyes, H.; Hallaq, B.; Cunningham, J.; Watson, T. The industrial internet of things (IIoT): An analysis framework. Comput. Ind. 2018, 101, 1-12. [CrossRef]

15. Serpanos, D.; Wolf, M. Industrial Internet of Things. In Internet-of-Things (IoT) Systems: Architectures, Algorithms, Methodologies; Springer: Cham, Switzerland, 2018; pp. 37-54. [CrossRef]

16. Hermann, M.; Pentek, T.; Otto, B. Design Principles for Industrie 4.0 Scenarios. In Proceedings of the 2016 49th Hawaii International Conference on System Sciences (HICSS), Koloa, HI, USA, 5-8 January 2016; pp. 3928-3937. [CrossRef]

17. Shahzad, K.; O’Nils, M. Condition Monitoring in Industry 4.0-Design Challenges and Possibilities: A Case Study. In Proceedings of the 2018 Workshop on Metrology for Industry 4.0 and IoT, Brescia, Italy, 16-18 April 2018; pp. 101-106. [CrossRef]

18. Tan, J.; Koo, S.G. A Survey of Technologies in Internet of Things. In Proceedings of the 2014 IEEE International Conference on Distributed Computing in Sensor Systems, Marina Del Rey, CA, USA, 26-28 May 2014; pp. 269-274. [CrossRef]

19. Leonardi, L.; Battaglia, F.; Lo Bello, L. RT-LoRa: A Medium Access Strategy to Support Real-Time Flows Over LoRa-Based Networks for Industrial IoT Applications. IEEE Internet Things J. 2019, 6, 10812-10823. [CrossRef]

20. Haxhibeqiri, J.; Karaagac, A.; Van den Abeele, F.; Joseph, W.; Moerman, I.; Hoebeke, J. LoRa indoor coverage and performance in an industrial environment: Case study. In Proceedings of the 2017 22nd IEEE International Conference on Emerging Technologies and Factory Automation (ETFA), Limassol, Cyprus, 12-15 September 2017; pp. 1-8. [CrossRef]

21. Lam, K.; Cheung, C.; Lee, W. LoRa-based localization systems for noisy outdoor environment. In Proceedings of the 2017 IEEE 13th International Conference on Wireless and Mobile Computing, Networking and Communications (WiMob), Rome, Italy, 9-11 October 2017; pp. 278-284. [CrossRef]

22. Savazzi, P.; Goldoni, E.; Vizziello, A.; Favalli, L.; Gamba, P. A Wiener-Based RSSI Localization Algorithm Exploiting Modulation Diversity in LoRa Networks. IEEE Sens. J. 2019, 19, 12381-12388. [CrossRef]

23. Singh, G.; Sundaram, K.; Saleh, A. Addressing reduced ingress protection class \& proper filter selection for open ventilated (IC3A1) wind turbine generator. In Proceedings of the 2019 10th International Renewable Energy Congress (IREC), Sousse, Tunisia, 26-28 March 2019; pp. 1-5.

24. Alvarez Paniagua, J.E. Mantenimiento Predictivo a Través de un Sistema de Monitoreo de Vibraciones a Turbinas Tipo Francis (8MW) Acorde a la Norma ISO 10816, en la Central Hidroeléctrica Santa Teresa. Ph.D. Thesis, Universidad de San Carlos de Guatemala, Guatemala City , Guatemala, 2019.

25. Flores, E.; Albornozb, A.; Lópezb, E.; Romeroc, J. Vibration assessment of transmission bearings of a mini Baja SAE prototype. Rev. Ing. UC 2020, 27, 41-53.

26. Dondurur, D. Chapter 4-Fundamentals of Data Processing. In Acquisition and Processing of Marine Seismic Data; Dondurur, D., Ed.; Elsevier: Amsterdam, The Netherlands, 2018; pp. 211-239. [CrossRef]

27. Song, Y.; Lin, J.; Tang, M.; Dong, S. An Internet of energy things based on wireless LPWAN. Engineering 2017, 3, 460-466. [CrossRef]

28. Augustin, A.; Yi, J.; Clausen, T.; Townsley, W.M. A study of LoRa: Long range \& low power networks for the internet of things. Sensors 2016, 16, 1466.

29. Adelantado, F.; Vilajosana, X.; Tuset-Peiro, P.; Martinez, B.; Melia-Segui, J.; Watteyne, T. Understanding the limits of LoRaWAN. IEEE Commun. Mag. 2017, 55, 34-40. [CrossRef] 
30. Bor, M.C.; Roedig, U.; Voigt, T.; Alonso, J.M. Do LoRa low-power wide-area networks scale? In Proceedings of the 19th ACM International Conference on Modeling, Analysis and Simulation of Wireless and Mobile Systems, Malta, Malta, 13-17 November 2016; pp. 59-67.

31. Torralba, M.; Adeva, P.; Lieblich, M.; Ibáñez, J.; Caruana, G.; García Escorial, A.; González-Doncel, G. Influencia del tamaño de partícula de polvo sobre las propiedades mecánicas de la aleación PM 7075 reforzada con SiCp. Available online: https://revistademetalurgia.revistas.csic.es/index.php/revistademetalurgia/article/download/774/785 (accessed on 15 November 2021).

32. Bloch, H.P. Ingress Protection code explained. World Pumps 2009, 2009, 26. [CrossRef]

33. Kendall, A.; Gal, Y. What uncertainties do we need in bayesian deep learning for computer vision? arXiv 2017, arXiv:1703.04977.

34. Arias Chao, M.; Kulkarni, C.; Goebel, K.; Fink, O. Fusing physics-based and deep learning models for prognostics. Reliab. Eng. Syst. Saf. 2022, 217, 107961. [CrossRef]

35. Mazaev, T.; Crevecoeur, G.; Hoecke, S.V. Bayesian Convolutional Neural Networks for Remaining Useful Life Prognostics of Solenoid Valves With Uncertainty Estimations. IEEE Trans. Ind. Inform. 2021, 17, 8418-8428. [CrossRef]

36. Peng, W.; Ye, Z.S.; Chen, N. Bayesian Deep-Learning-Based Health Prognostics Toward Prognostics Uncertainty. IEEE Trans. Ind. Electron. 2020, 67, 2283-2293. [CrossRef]

37. Cushing, M.J.; Mortin, D.E.; Stadterman, T.J.; Malhotra, A. Comparison of electronics-reliability assessment approaches. IEEE Trans. Reliab. 1993, 42, 542-546. [CrossRef]

38. Muhammad, N.; Fang, Z.; Shah, S.Y.; Haider, D. Reliability and remaining life assessment of an electronic fuze using accelerated life testing. Micromachines 2020, 11, 272. [CrossRef] [PubMed] 